\title{
Special Issue on Liquid Crystal on Silicon Devices: Modeling and Advanced Spatial Light Modulation Applications
}

\author{
Andrés Márquez ${ }^{1,2, *(1)}$ and Ángel Lizana ${ }^{3, *}$ \\ 1 Instituto Universitario de Física Aplicada a las Ciencias y las Tecnologías (IUFACyT), \\ Universidad de Alicante, 03690 Alicante, Spain \\ 2 Departamento de Física, Ingeniería de Sistemas y Teoría de la Señal, Universidad de Alicante, Ap. 99, \\ 03080 Alicante, Spain \\ 3 Departamento de Física, Universitat Autònoma de Barcelona, 08193 Bellaterra, Spain \\ * Correspondence: andres.marquez@ua.es (A.M.); angel.lizana@uab.es (Á.L.)
}

Received: 16 July 2019; Accepted: 26 July 2019; Published: 29 July 2019

\section{Introduction}

Since the first liquid crystal displays (LCDs) at the beginning of the seventies-based on the twisted-nematic cell configuration [1] — LC-based devices [2] have shown a great potential not only as a display technology, but also for spatial light modulation applications.

Among the different LC-based technologies, liquid crystal on silicon (LCoS) has become one of the most widespread technologies for spatial light modulation in optics and photonics' applications [3-6]. These reflective microdisplays are composed of a high-performance silicon complementary metal oxide semiconductor (CMOS) backplane, which controls the liquid crystal layer's light modulating properties . State-of-the-art LCoS microdisplays may exhibit a very small pixel pitch (below $4 \mu \mathrm{m}$ ), a very large number of pixels (resolutions larger than $4 \mathrm{~K}$ ), and high fill factors (larger than 90\%). They modulate illumination sources covering the UV, visible, and IR.

LCoS technologies are used as displays as well as polarization, amplitude, and phase-only spatial light modulators, where they achieve full phase modulation. Due to their excellent modulating properties and high degree of flexibility, they are found in all sorts of spatial light modulation applications, such as in LCoS-based display systems for augmented and virtual reality, head-up display, head-mounted display, projector, true holographic displays, digital holography, optical storage, adaptive optics, diffractive optical elements, super-resolution optical systems, optical metrology techniques, reconfigurable interconnects, beam-steering devices, wavelength selective switches in optical telecommunications, wave-front sensing of structured light beams, holographic optical traps, or quantum optical computing.

In order to fulfill the requirements in this extensive range of applications, specific models and characterization techniques are proposed. These devices may exhibit a number of degradation effects such as limited modulation range for high spatial frequency image content, interpixel cross-talk and fringing field, and time flicker, which may also depend on the analog or digital backplane of the corresponding LCoS device. Appropriate characterization and compensation techniques are then necessary.

\section{Special Issue Papers}

This special issue provides a collection of papers demonstrating the impact of LCoS microdisplays in current and future spatial light modulation applications. State-of-the-art in LCoS device technology, LC materials, modeling and characterization techniques are presented. 
A complete review of LCoS origins, evolution, and applications is given by Chen et al. [7]. They cover both the interest in display applications and their developments as spatial light modulators (SLM), with special focus on phase-only spatial light modulation capabilities in digital holography or holographic video projection. The authors explain that the origin of LCoS backplane dates back to 1973, and the development of LCoS has been beneficial for full high definition displays and spatial light modulation. They start summarizing state-of-the-art developments of high-resolution panels, followed by addressing issues related to the driving frequency (i.e., liquid crystal response time and hardware interface), taking into account phase linearity control, phase precision, phase stability, and phase accuracy.

A good complement to the previous paper is given by Li and Cao [8]. The authors focus their review on the available characterization techniques to obtain the grayscale-phase response of LCoS devices. They demonstrate that precise calibrations are necessary since they greatly influence the results when applied in phase-only applications, including the majority of interesting cases such as holographic display, optical tweezers, lithography, etc. Due to limitations in the manufacturing process, the grayscale-phase response could be different for every single SLM device, even varying on sections of an SLM panel due to the screen or electrical addressing spatial inhomogeneities. They divide the numerous calibration methods into two categories: the interferometric phase calibration methods and the diffractive phase calibration methods. The main phase calibration methods are discussed and reviewed, comparing their possible advantages in different applications.

Regarding the analysis of LC materials enabled for LCoS devices, we consider the review paper by Andreev et al. [9]. They investigate ferroelectric liquid crystals (FLCs) of a new type developed for fast low-voltage displays and light modulators. They are helix-free FLCs, characterized by spatially periodic deformation of smectic layers and a small value of spontaneous polarization. Both theoretical models and experimental results are presented for modulation of light transmission, scattering, and phase delay with a high rate.

Alternative optically addressable spatial-light modulators (OASLM) are investigated by Pei et al. [10], which are very interesting for phase-only modulation applications. They focus on the influence of driving conditions on their phase-modulation ability. To this end, they use an equivalent circuit method and a system for measuring wave-front modulation that uses a phase-unwrapping data-processing method, and is constructed with a charge-coupled device and wave-front sensor. They demonstrate that wave-front on-line modulation with feedback control is possible with the OASLM and the corresponding monitoring system.

In previous years, there has been considerable interest in augmented reality (AR) displays. Huang et al. [11] review LCoS technology capabilities' new application in emerging AR displays. They start by reviewing the LCoS working principles of three commonly adopted LC modes. Then, the fringing field effect is analyzed, which is very important in very high resolution LCoS microdisplays. The novel pretilt angle patterning method for suppressing the effect is presented. They also show how to integrate the LCoS panel in an AR display system. Authors show that the application of LCoS in AR head-mounted displays and head-up displays is foreseeable.

In dealing with holographic projection and application of phase-only holography with SLMs for display applications, we consider the paper by Christmas and Collings [12]. They review the various approaches of producing dynamic holographic displays and show their particular proposal with a superior light efficiency and fault tolerance. Holographic displays favor small pixel devices, with LCoS devices as optimum candidates for these applications to become commercially feasible.

Davis et al. [13], in imaging applications, demonstrate a programmable zoom lens system where the magnification and sense of the image can be controlled without moving any parts. They use two programmable SLMs onto which they encode the required focal length lenses to achieve these results. They show both theoretical calculations and experimental results. The authors discuss the system's size limitations caused by the limited spatial resolution, and show how newer devices-LCoS with a very high resolution-would shrink the size of the system. 
SLMs are also used in holographic data storage systems (HDSS). The paper by Martínez-Guardiola et al. [14] presents a method to characterize a complete optical HDSS. In this study, the authors identify the elements that limit the capacity to register and restore the information introduced by means of a LCoS microdisplay as the data pager. They further test whether the anamorphic and frequency-dependent effect is relevant in the application to HDSS, where nonperiodic binary elements are applied. They consider anamorphic patterns with different resolutions addressed to the LCoS. They show both the precharacterization results when no recording material is in the HDSS and when introducing a photopolymer as the recording material.

Another interesting application for LCoS devices is described by Rothe et al. [15]. Multimode fibers (MMF) are promising candidates for increasing the data rate while reducing the space required for optical fiber networks. The authors demonstrate a method for measuring the transmission matrix (TM) of a multimode fiber. It is based on mode-selective excitation of complex amplitudes performed with only one phase-only spatial light modulator. The light field propagating through the fiber is measured holographically and analyzed by a rapid decomposition method. The TM determines the amplitude and phase relationships of the modes, allowing us to understand the mode scrambling processes in the MMF, which can also be used for mode division multiplexing in telecommunications.

In the review paper by Zhang et al. [16], the authors demonstrate an LCoS self-calibration technique, from which they perform a complete LCoS characterization. They determine its phase-voltage curve by using the interference pattern generated by a digital two-sectorial split-lens configuration. They also determine the LCoS surface profile by using a self-addressed dynamic microlens array pattern. Once the LCoS is calibrated, they show both the application to microparticle manipulation through light optical traps created by a LCoS display. They also show the ability of the LCoS display to implement a holographic imaging system with a double-sideband filter configuration, so as to obtain dynamic holographic imaging of microparticles.

In the last paper of this collection, Pérez-Cabré and Millán [17] characterize a LCoS with a phase modulation much larger than $2 \pi$ radians. Multiorder diffractive optical elements, displayed on the LCOS SLM with the appropriate phase modulation range, enable the design and experimental demonstration of an achromatic multiorder lens. They show that the residual chromatic aberration is reduced to one-third that of the chromatic aberration of a conventional first-order diffractive lens.

\section{Perspectives}

This Special Issue contains eleven papers addressing a wide variety of topics dealing with liquid crystal on silicon (LCoS) devices. The review and research papers provide a good insight into the present developments in this topic. We note that the availability of more mature technological devices, with ever decreasing pixel sizes and more stable operation, indicate that LCoS will widen their range of applicability even more in the coming years.

Funding: This research received no external funding.

Acknowledgments: The guest editors would like to thank all the authors and reviewers for contributing with their excellent papers and outstanding evaluation reports to this special issue. The guest editors also would like to thank the MDPI team involved in editing and managing this special issue. We would also like to thank Lucia Li, the contact editor for this special issue, for her very professional and generous support.

Conflicts of Interest: The authors declare no conflict of interest.

\section{References}

1. Schadt, M.; Helfrich, W. Voltage-dependent optical activity of a twisted nematic liquid crystal. Appl. Phys. Lett. 1971, 18, 127-128. [CrossRef]

2. Yeh, P.; Gu, C. Optics of Liquid Crystal Displays, 2nd ed.; Wiley: Hoboken, NJ, USA, 2009.

3. Collings, N.; Davey, T.; Christmas, J.; Chu, D.; Crossland, B. The Applications and Technology of Phase-Only Liquid Crystal on Silicon Devices. J. Disp. Technol. 2011, 7, 112-119. [CrossRef] 
4. Lazarev, G.; Hermerschmidt, A.; Kruger, S.; Osten, S. LCoS Spatial Light Modulators: Trends and Applications, in Optical Imaging and Metrology: Advanced Technologies; Osten, W., Reingand, N., Eds.; Wiley-VCH Verlag \& Co.: Weinheim, Germany, 2012. [CrossRef]

5. Zhang, Z.; You, Z.; Chu, D. Fundamentals of phase-only liquid crystal on silicon (LCOS) devices. Light Sci. Appl. 2014, 3, e213. [CrossRef]

6. Lazarev, G.; Chen, P.-J.; Strauss, J.; Fontaine, N.; Forbes, A. Beyond the display: Phase-only liquid crystal on Silicon devices and their applications in photonics. Opt. Express 2019, 27, 16206-16249. [CrossRef] [PubMed]

7. Chen, H.-M.P.; Yang, J.-P.; Yen, H.-T.; Hsu, Z.-N.; Huang, Y.; Wu, S.-T. Pursuing High Quality Phase-Only Liquid Crystal on Silicon (LCoS) Devices. Appl. Sci. 2018, 8, 2323. [CrossRef]

8. Li, R.; Cao, L. Progress in Phase Calibration for Liquid Crystal Spatial Light Modulators. Appl. Sci. 2019, 9, 2012. [CrossRef]

9. Andreev, A.; Andreeva, T.; Kompanets, I.; Zalyapin, N. Helix-Free Ferroelectric Liquid Crystals: Electro Optics and Possible Applications. Appl. Sci. 2018, 8, 2429. [CrossRef]

10. Pei, L.; Huang, D.; Fan, W.; Cheng, H.; Li, X. Phase-Only Optically Addressable Spatial-Light Modulator and On-Line Phase-Modulation Detection System. Appl. Sci. 2018, 8, 1812. [CrossRef]

11. Huang, Y.; Liao, E.; Chen, R.; Wu, S.-T. Liquid-Crystal-on-Silicon for Augmented Reality Displays. Appl. Sci. 2018, 8, 2366. [CrossRef]

12. Christmas, J.; Collings, N. Displays Based on Dynamic Phase-Only Holography. Appl. Sci. 2018, 8, 685. [CrossRef]

13. Davis, J.A.; Hall, T.I.; Moreno, I.; Sorger, J.P.; Cottrell, D.M. Programmable Zoom Lens System with Two Spatial Light Modulators: Limits Imposed by the Spatial Resolution. Appl. Sci. 2018, 8, 1006. [CrossRef]

14. Martínez-Guardiola, F.J.; Márquez, A.; Calzado, E.M.; Bleda, S.; Gallego, S.; Pascual, I.; Beléndez, A. Anamorphic and Local Characterization of a Holographic Data Storage System with a Liquid-Crystal on Silicon Microdisplay as Data Pager. Appl. Sci. 2018, 8, 986. [CrossRef]

15. Rothe, S.; Radner, H.; Koukourakis, N.; Czarske, J.W. Transmission Matrix Measurement of Multimode Optical Fibers by Mode-Selective Excitation Using One Spatial Light Modulator. Appl. Sci. 2019, 9, 195. [CrossRef]

16. Zhang, H.; Lizana, A.; Van Eeckhout, A.; Turpin, A.; Ramirez, C.; Iemmi, C.; Campos, J. Microparticle Manipulation and Imaging through a Self-Calibrated Liquid Crystal on Silicon Display. Appl. Sci. 2018, 8, 2310. [CrossRef]

17. Pérez-Cabré, E.; Millán, M.S. Liquid Crystal Spatial Light Modulator with Optimized Phase Modulation Ranges to Display Multiorder Diffractive Elements. Appl. Sci. 2019, 9, 2592. [CrossRef] 